\title{
PIV investigation on the turbulence in a gas-liquid separator
}

\author{
Junlian yin ${ }^{1}$, Tingting Zhang ${ }^{2}$, Guangyuan Huang ${ }^{2}$, and Dezhong Wang ${ }^{2}$ \\ ${ }^{1}$ Shanghai Jiao Tong University \\ ${ }^{2}$ Affiliation not available
}

May 5, 2020

\begin{abstract}
The two-phase flow in an axial gas-liquid separator is critical for the gas separation in the thorium molten salt reactor. Although the two-phase flow pattern is visualized and the velocity field is obtained in our previous studies, the turbulence field is still unknown. To clarify the statistics of the turbulence field, we used the stereo PIV technique to explore the instantaneous fluctuations of three velocity components. The turbulence information including the turbulent kinetic energy and three typical Reynolds shear stresses were extracted from five typical planar positions covering the whole swirl chamber. The results indicate that the distribution of turbulence statistics presents a very different tendency of distribution, as compared to conventional cyclones. The difference is distinguished by the varying anisotropic stress field and the double peak distribution of turbulent kinetic energy. The turbulence data provides a complete and reliable foundation for two-phase turbulence modeling.
\end{abstract}

\section{Hosted file}

Part 2 Turbulence statistics Gas-Liquid Separator.docx available at https://authorea. com/users/292611/articles/420315-piv-investigation-on-the-turbulence-in-a-gas-liquidseparator 